\title{
Multiple Pre-Pyloric Gastric Diverticula
}

\author{
Stephen J Mularz*, Christopher Chum and Michael David Bernstein \\ Coney Island Hospital Department of Gastroenterology, USA
}

Submission: June 26, 2020; Published: August 07, 2020

*Corresponding author: Stephen J Mularz, Coney Island Hospital Department of Gastroenterology, 2612 West 2nd St. \#1F, 2601 Ocean PKWY, Brooklyn, NY 11223, USA

Abstract

Gastric Diverticulum is a rare but well-described entity. We present here an incidentally-found set of three tightly-grouped, mediumsized pre-pyloric gastric diverticula discovered in a 51 year-old woman undergoing upper endoscopy to evaluate ongoing GERD and gastritis symptoms. Our case of multiple medium-sized pre-pyloric diverticula is likely extremely rare.

Keywords: Diverticulum; Diverticula; Diverticula; Gastric Diverticula; Antrum; Endoscopy

\section{Introduction}

Gastric Diverticulum is a rare but well-described entity. A cursory literature review provides the statistics and hypothesized etiologic processes involved. One review by Farhan Rashid et al. and another brief review that also includes two case reports by Luigi Marano et al. are excellent sources [1,2].

The majority of published case reports provide multiple images of singular, incidentally found gastric diverticula as photographed endoscopically or as seen with barium and/or CT studies.

\section{Case Report}

We present here an incidentally-found set of three tightlygrouped, medium-sized pre-pyloric gastric diverticula discovered in a 51 year-old woman undergoing upper endoscopy to evaluate ongoing GERD and gastritis symptoms. Her medical history was significant for multiple antral gastric ulcers "many years ago" found on endoscopy in Ukraine. Prior H. pylori status is not known and current testing is pending at the time of this writing (Figure 1).

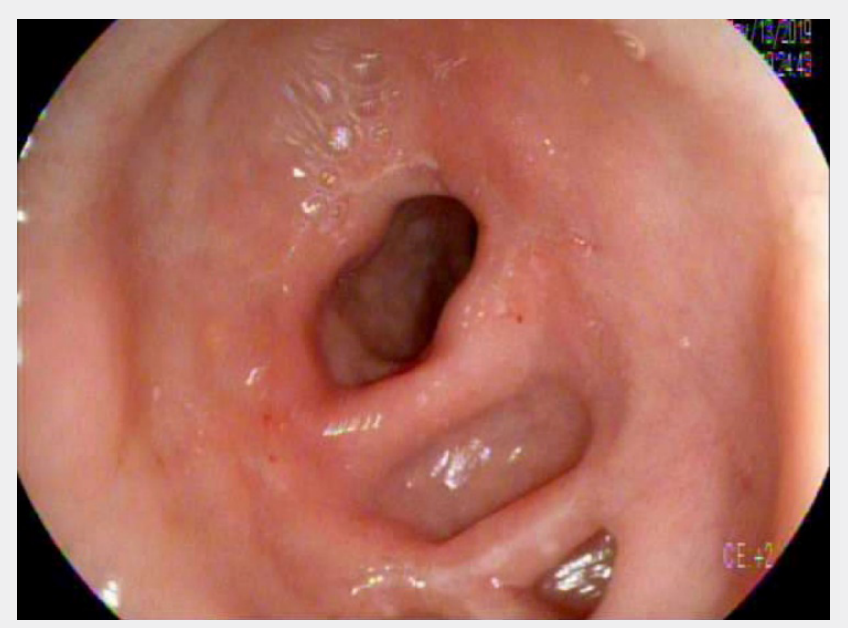

Figure 1: Two of the three pre-pyloric gastric diverticula are seen below the true pylorus. 
Gastric diverticulum is rare, it is incidentally found radiologically at a rate of $0.04 \%$ and endoscopically in $0.11 \%$ $[3,4]$. "True" (involving all gastric layers) congenital diverticula are more common than their acquired "false" cousins. About three quarters of the true diverticula reside in the posterior wall of the fundus, $2 \mathrm{~cm}$ below the GE junction [5]. Pseudo-diverticula are less commonly known, but they do tend to occur in the antrum. These false diverticula usually arise from inflammatory processes such as ulcers, prior bariatric surgeries or increased wall tension due to gastric outlet obstruction and/or excessive vomiting [6] (Figure 2).

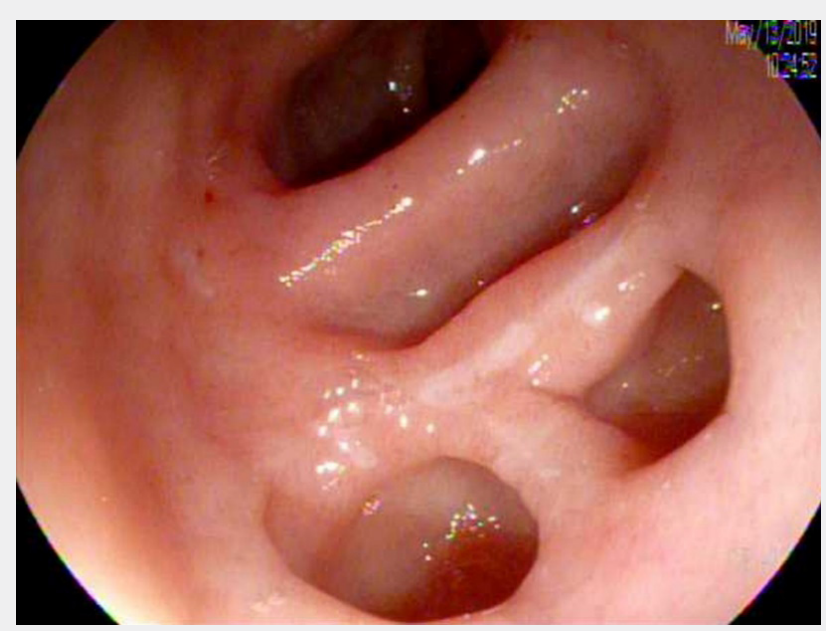

Figure 2: Three grouped antral diverticula seen just below the pylorus.

Our case of multiple medium-sized pre-pyloric diverticula is likely extremely rare-this author could not find evidence of prior published case reports of multiple coexisting pre-pyloric diverticula. There are no specific symptoms to alert the clinician to the presence of a gastric diverticulum. Most are asymptomatic but when they are symptomatic, they can mimic other common upper GI diseases such as gastritis [7]. Congenital diverticula may even contain ectopic pancreas [5,8].

\section{Discussion}

No treatment is required for a modest-sized and incidentally found, asymptomatic diverticulum. If symptoms are present, such as gastritis-like complaints, PPI therapy should be utilized [7]. More drastic sequelae will require surgical resection (commonly via laparoscopic diverticulotomy). Surgical therapy should be reserved for those with bleeding, perforation, malignancy or distressing symptoms or pain that is refractory to PPI therapy. Surgery is also recommended to correct very large diverticularegardless of symptoms.

Though gastric diverticula are rare, they are encountered. If a patient has no other endoscopic explanation for ongoing symptoms, the diverticulum may be to blame. Surgical correction is available, well-tolerated and safe. Our patient is undergoing therapy with PPI again. If this does not help resolve her GERD and gastritis-like symptoms, her future therapy will involve a surgical referral.

\section{References}

1. Rashid F, Aber A, Iftikhar SY (2012) A review on gastric diverticulum. World J Emerg Surg 7(1): 1.

2. Marano L, Reda G, Porfidia R, Michele Grassia, Marianna Petrillo, et al (2013) Large symptomatic gastric diverticula: two case reports and a brief review of literature. World J Gastroenterol 19(36): 6114-6117.

3. Gockel I, Thomschke D, Lorenz D (2004) Gastrointestinal: Gastric diverticula. Journal of Gastroenterology and Hepatology 19: 227-227.

4. Schiller AH1, Roggendorf B, Delker-Wegener S (2007) Laparoscopic resection of gastric diverticula: two case reports. Zentralbl Chir 132(3): 251-255

5. Wolters VM1, Nikkels PG, Van Der Zee DC (2001) A gastric diverticulum containing pancreatic tissue and presenting as congenital double pylorus: case report and review of the literature. J Pediatr Gastroenterol Nutr 33(1): 89-91.

6. Meeroff M, Gollan JR, MEeroff JC (1967) Gastric Diverticulum. Am J Gastroenterol 47: 189-203.

7. Mohan P, Ananthavadivelu, Venkataraman J (2010) Gastric Diverticulum. CMAJ 182(5): 226.

8. Hewa TL, Zhang Z, Rozelle C, Annie Terry (2011) Gastric antral diverticulum with heterotopic pancreas in a teenage patient. JPGN 53(5): 471. 
